\title{
The EUSO Data Simulation and Analysis Tree
}

\author{
Maria Concetta Maccarone \\ INAF/IASF-Palermo, Istituto di Astrofisica Spaziale e Fisica Cosmica \\ Via Ugo La Malfa 153, 90146 Palermo, Italy \\ Cettina.Maccarone@pa.iasf.cnr.it \\ Giacomo D'Alì Staiti \\ DIFTER Dept. of Physics, University of Palermo, and INAF/IASF-Palermo \\ Via Ugo La Malfa 153, 90146 Palermo, Italy \\ Giacomo.Dali@pa.iasf.cnr.it \\ Sergio Bottai \\ Dept. of Physics, University of Florence \\ Via G. Sansone 1, 50019 Sesto Fiorentino, Firenze, Italy \\ Bottai@fi.infn.it \\ Marco Pallavicini \\ INFN-Genova, Istituto Nazionale di Fisica Nucleare \\ Via Dodecaneso 33, 16146 Genova, Italy \\ Marco.Pallavicini@ge.infn.it \\ Alessandro Thea \\ Dept. of Physics, University of Genova \\ Via Dodecaneso 33, 16146 Genova, Italy \\ Alessandro.Thea@ge.infn.it
}

\begin{abstract}
The "Extreme Universe Space Observatory - EUSO" is the first Space mission devoted to the exploration of the outermost bounds of the Universe through the investigation of the Extremely-High Energy Cosmic Rays, EECR, using the Earth atmosphere as a giant detector. The objective is to obtain a detailed description of the Cosmic Ray spectrum beyond $5 \times 10^{19} \mathrm{eV}$ together with a map of the arrival directions. EUSO will detect EECR looking at the streak of fluorescence light produced when such a particle interacts with the atmosphere. The signal will be detected after its propagation upward from the dark Earth atmosphere to the EUSO telescope accommodated, as external payload, on the International Space Station. EUSO is a mission of the European Space Agency ESA, and it is now completing the "Phase A" study with a goal for a three year mission starting in 2010.
\end{abstract}

The various peculiarities of the EUSO space-based observational approach imply a dedicated effort for the evaluation of the expected features of the detected signals and for the reconstruction of its spacetime development, energy and composition, namely from simulation and data analysis point of view. A complete software infrastructure named ESAF (EUSO Simulation and Analysis Framework) is under development suitable for event simulation, detector response simulation, event reconstruction and scientific data analysis.

EUSO - Extremely High Energy Cosmic Ray - Data Simulation - Data Analysis - Software System - ESAF.

\section{INTRODUCTION}

Cosmic Rays are particles which constantly bombard the Earth travelling at nearly the speed of light and arriving from all the directions of the outer Space. The flux of Cosmic Rays (CR) reaching the Earth falls rapidly with energy, decreasing by a factor 50 to 100 for each decade of increasing energy. The CR energy spectrum starts from $\sim 10^{9} \mathrm{eV}$ and continues up to $10^{20} \mathrm{eV}$ and beyond. The observation of Extremely-high Energy Cosmic Rays (EECR) and neutrinos (EEv) is a very challenging issue: in fact, the existence of sub-atomic particles with individual 
energy greater than $5 \times 10^{19} \mathrm{eV}$ (where the so-called GZK cut-off is expected to take place) raises fundamental scientific questions in connection with their origin and propagation in the interstellar and intergalactic space. Where do EECR particles come from? What is the EECR maximum energy, if any? What is their composition? What is their propagation mechanism? ... these are only few of the many questions which have not yet received a definitive or satisfying answer.

Roughly below $10^{15} \mathrm{eV}$ Cosmic Rays can be measured in a direct way. Above this energy, the CR flux decreases drastically (from 1 particle/( $\mathrm{m}^{2}$ year) at $\sim 10^{15} \mathrm{eV}$ to 1 particle/( $\mathrm{km}^{2}$ century) at $\sim 10^{20} \mathrm{eV}$ ) and direct measurements are no more feasible. But, fortunately, at these energies a Primary CR, colliding with air nuclei in the atmosphere, produces secondaries that in turn collide with air atoms giving rise to a propagating cascade of particles, the Extensive Air Showers (EAS): it is through the observation of EAS that is possible to measure, indirectly, the highenergy Primary Cosmic Ray. Ground-based experiments used and use different techniques to achieve the goal that is a very challenging task: the result of more than forty years of observation is that only about twenty of EECR with energy greater than $10^{20} \mathrm{eV}$ have been observed.

One of the various observation techniques is related to the fluorescence induced in air Nitrogen by EAS. The most numerous particles in an EAS are electrons; their number at shower maximum development is proportional to the energy of the Primary. Electrons moving through the atmosphere ionise the air and excite metastable energy levels in its atoms and molecules. With a short relaxation time, electrons from those levels return to ground state emitting a characteristic fluorescence light. In air the fluorescence extends from Infrared to Ultraviolet (UV), with peaks at wavelengths from $330 \mathrm{~nm}$ to $450 \mathrm{~nm}$. The emitted light is isotropic and proportional to the shower energy at any given depth in the atmosphere. Moreover, highly collimated Čerenkov photons are produced along the shower axial direction, too. A high energy EAS forms a significant streak of fluorescence light over 10-100 km in length along its passage in the atmosphere, depending on the energy of the Primary and the angle with the vertical; observation of the trajectory of an EAS through the atmosphere then allows reconstruction of the trajectory and determination of the full cascade development. To date, the only ground-based experiments to implement this technique are Fly's Eye [1] and its successor HiRes [2] (observing the North sky), and part of the Auger Observatory [3], the biggest world observatory now under construction in Argentina (South sky). The Fly's Eye reported the highest EECR event observed up to date, with an energy of $3 \times 10^{20} \mathrm{eV}[4]$.

But the fluorescence light is not so big: its feeble flare can be compared with a $100 \mathrm{~W}$ UV lamp travelling at the speed of light in the atmosphere. To observe the UV fluorescence light it is then necessary a fast and sensitive electronics, night observations at clear sky (better if moonless) to improve efficiency, absence of light pollution. Moreover the expected flux of EECR above $10^{20} \mathrm{eV}$ is about 1 particle/( $\mathrm{km}^{2}$ century). As firstly suggested by J. Linsley [5], who was also the first to claim detection of a $10^{20} \mathrm{eV}$ event, a solution to improve statistics is provided by observing from Space the UV induced fluorescence in the Earth's atmosphere, which allows exploiting a more bigger acceptance area with respect to the ground-based observatories, obtaining an all-sky (North and South) coverage, and also observing the reflected UV light due to the impact of the Čerenkov beam, accompanying the EAS, on top of clouds, land or sea.

EUSO, the "Extreme Universe Space Observatory" $[6,7,8,9]$ is the first experiment from Space devoted to the investigation of the EECR/v radiation using the Earth's atmosphere as a "giant detector". EUSO is a mission of the European Space Agency, ESA; the closure of its Phase A study is expected by ESA within July 2004. EUSO will observe the fluorescence and Čerenkov signals looking downward the dark Earth atmosphere being accommodated as external payload onboard the International Space Station, ISS. According to the current ISS Launch Manifest now under definition, the launch of EUSO is scheduled in 2010 for a three year mission.

The various peculiarities of the EUSO space-based observational approach imply a dedicated effort for the evaluation of the expected features of the detected signals and for the reconstruction of its space-time development, energy and composition. The goal is that of obtaining an end-to-end simulation chain finalised to study the detector response function [8]. During Phase A study, the aim was that of identifying the problems, develop the algorithm to treat them and derive the expected detector performances. A complete software infrastructure named ESAF, EUSO Simulation and Analysis Framework, [10, 11] is now under development suitable for event simulation, detector response simulation, event reconstruction and scientific data analysis.

Aim of this paper is to present the EUSO data simulation, reconstruction and analysis tree, with some notes about the problems encountered in defining it, together with the general structure of the ESAF system. An overview of EUSO and of its observation operative mode is given firstly (for more scientific and technical details, see References [8, 9]). 


\section{EUSO}

\subsection{Instrument Overview and Observational Approach}

The EUSO main EECR/v telescope presents a compact instrument design: a couple of large Fresnel lenses $(\sim 2.5$ $\mathrm{m} \varnothing)$ forms the optics system which addresses the received UV light onto a finely segmented focal surface composed by fast detectors (Multi Anode Photo Multiplier Tube, MAPMT, 10 ns resolution time) operating in the UV band $330 \div 400 \mathrm{~nm}$. The fast and modular read-out electronics system registering the signal is able to handle more than 250000 channels, the total of pixels composing the MAPMT focal detector, and it is comprehensive of a dedicated track recorder acting as a trigger. All these parts are assembled together in an envelope, as sketched in Fig.1-a. The wide-angle field of view (FoV) of EUSO $\left( \pm 30^{\circ}\right)$ combined with the altitude of the telescope accommodated on the ISS (of the order of $400 \mathrm{~km}$ ) (see Fig.1-b) will allow EUSO to observe a portion of $\sim 230 \mathrm{~km}$ radius of Earth surface, as sketched in Fig.1-c, corresponding to a target mass of $\sim 2 \times 10^{12}$ tons of atmosphere.

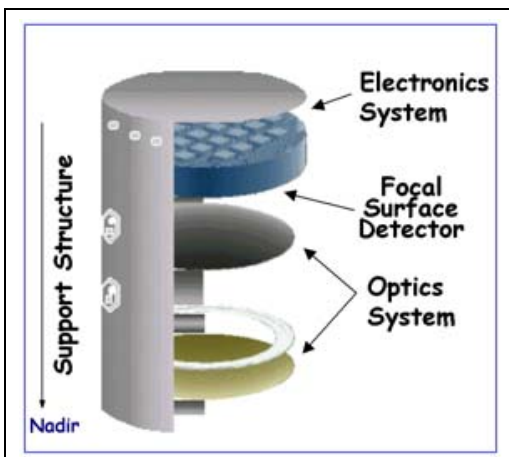

(a)

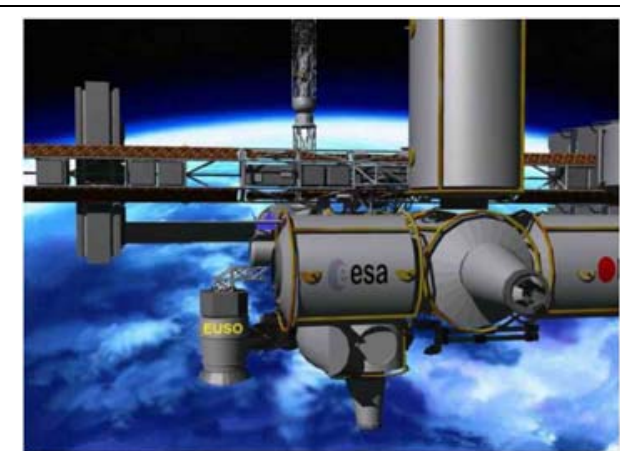

(b)

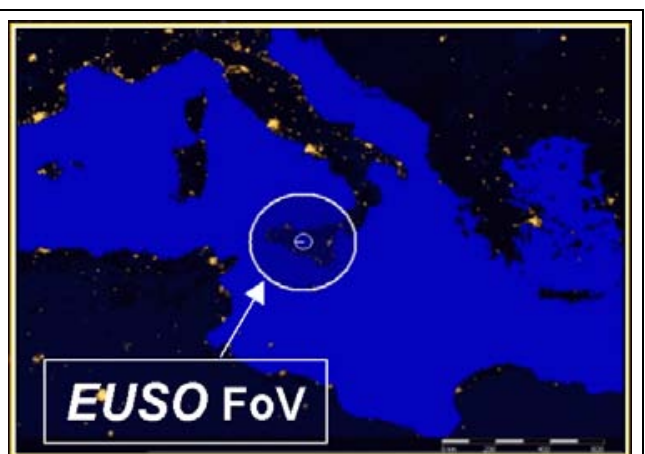

(c)

FIGURE 1: The EUSO main EECR/v telescope: (a) general scheme; (b) the telescope accommodated on the ISS; (c) the EUSO field of view on the Earth surface

The EUSO observational approach is based on the detection of the isotropic UV fluorescence light emitted by EAS along its passage trough the Earth's atmosphere, and of the diffuse reflected UV light due to the impact of the Čerenkov beam, accompanying the EAS, on clouds, land or sea. The Earth's atmosphere is the "natural" primary detector for EECR/v and EUSO uses the atmosphere as the interaction region of a Gas Counter where UV fluorescence light is produced. But, differently from an experimental Gas Scintillation Counter produced in lab, all the atmosphere parameters can only be measured: they cannot be modified. Moreover, since the ISS covers the whole Earth surface in the latitude range $\pm 51^{\circ}$ and moves at a speed of $\sim 7 \mathrm{~km} / \mathrm{s}$, the variability of the scene seen by EUSO in its FoV is high. It is therefore necessary to monitor the atmosphere at the trigger time, i.e. when a candidate EECR/v signal is registered by the EUSO electronics. The sounding of the atmosphere inside the FoV is performed, on an event-by-event basis, by a system coupled to the EUSO main telescope and consisting of an InfraRed (IR) camera (which allows a photograph of the clouds status at the moment of the trigger) and of a Lidarbased device (which will sound atmosphere and its properties along the EAS signal direction). The EUSO operative mode of observation can be schematised as follows (see Fig.2):

0. The EECR primary particle, interacting with the Earth's atmosphere, give rise to propagating Extensive Air Shower (EAS) of secondary particles accompanied by isotropic emission of UV fluorescence induced in air Nitrogen as result of a complex relativistic cascade process. An EAS corresponding to a Primary with energy $-10^{20} \mathrm{eV}$ forms a significant streak of fluorescence light over 10-100 km along its passage in the atmosphere, depending on the nature of the Primary and on the pitch angle with the vertical.

1. The isotropic UV fluorescence light is registered by the EECR/v telescope.

2. Highly collimated Čerenkov photons are produced along the shower axis. When the Čerenkov beam impacts on the top of a cloud, or on the sea or land, its reflected UV light is seen by the EECR/v telescope.

3. The EECR/v telescope continuously registers and, whenever the signal intensity is greater than a proper threshold, the electronics system understands that the signal could be the "good" one and then "triggers". A photograph of the FoV is then taken by the IR camera at the trigger time.

4. The atmosphere sounding is at the end performed by the Lidar device along the shower direction. 

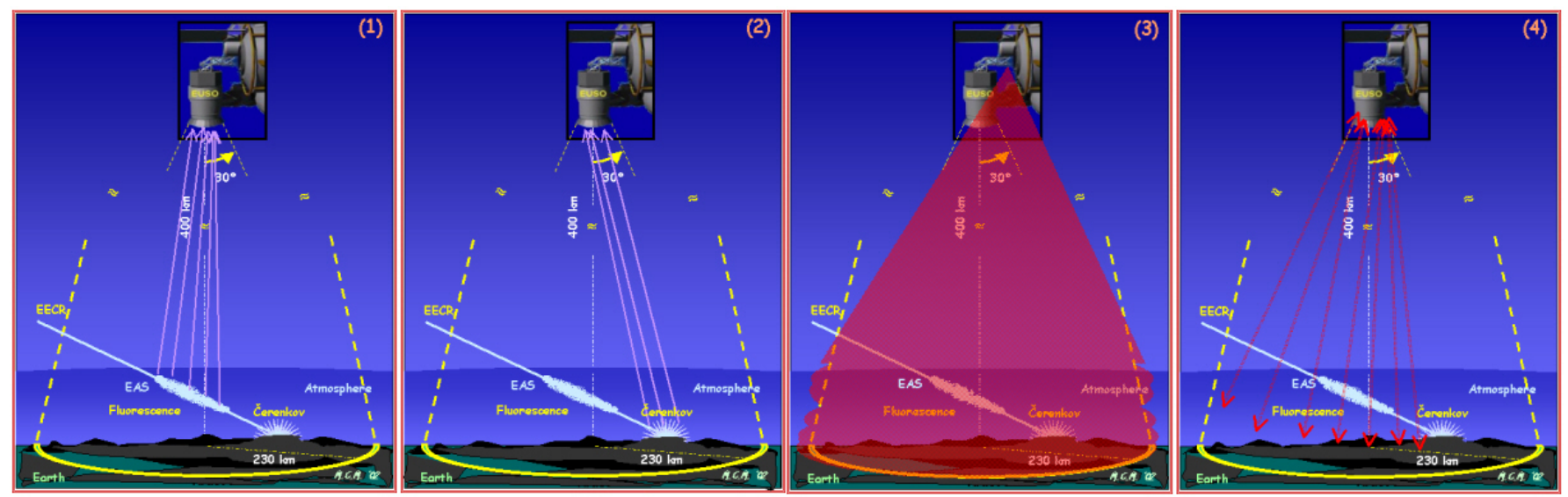

FIGURE 2: The EUSO observational operative mode (from left to right, see text)

By using atmosphere as a natural detector, EUSO will systematically survey a large portion of space providing a complete dataset of measurement in the $330 \div 400 \mathrm{~nm}$ band. Besides the EECR/v events, this dataset will contain information about all atmospheric UV-phenomena that occurred during the observation time (meteors, elves, lightning, ...). Moreover, it is mandatory to take into account all the background sources (natural or man-made) which can be seen inside the FoV and can distort or hide the "good" signal [12, 13].

\subsection{The End-To-End Simulation Chain for EUSO}

The various peculiarities of the EUSO space-based observational approach imply a dedicated effort for the evaluation of the expected features of the detected signal and for the reconstruction of its space-time development, energy and composition. First goal was to obtain an end-to-end simulation chain (as schematised in Fig.3) finalised to study the detector response function.

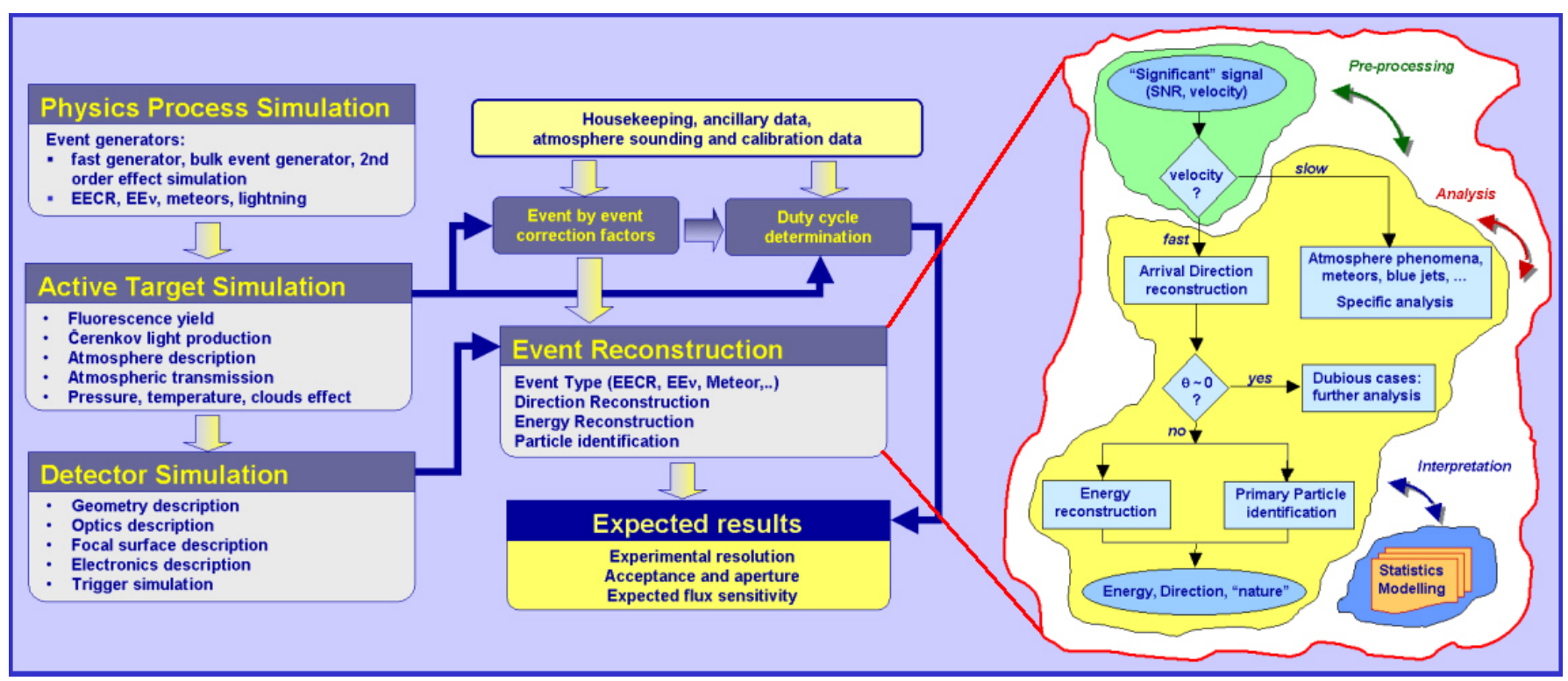

FIGURE 3: The general scheme of the End-To-End simulation chain for EUSO.

In this chain, three main simulation's tasks are needed to be accomplished: the physics process, the active target, and the detector, too. Each of them presents scientific and technical implementation problems.

Concerning the physics process, a proper EAS event generator must be developed. The most common and sophisticated MonteCarlo programs (CORSIKA [14], AIRES [15]) provide a detailed description but paid with a very high computing time, even in their "thinning" versions. In the EUSO chain, we have opted for, and firstly implemented, a simulation focused on the correct treatment of the longitudinal EAS profile, the fluorescence yield and Čerenkov light emission with less emphasis on the tracking of all the secondary particles produced in the 
shower (UNISIM [16], GIL [17, 18], SLAST [19]). At each relevant step of the generation process, a cross-check of the results with CORSIKA was however performed.

The simulation of the "active" target refers to the Earth's atmosphere which plays a twofold major role in EUSO. It is the light emission medium (yield of fluorescence from Nitrogen, and associated Čerenkov light), and the transmission medium where the light (fluorescence, diffuse and reflected Čerenkov UV light) propagates and attenuates from its source location to the telescope site. In the atmosphere seen as a light transmission medium, the fluorescence yield mainly depends on the EECR/v energy, on local properties of the atmosphere (air density, temperature, humidity) as well as on the altitude on which the EAS develops. At the same time, the production of the Čerenkov light accompanying the EAS is a function of the energy and of the air refraction index. The transmission of the photons from their source location to the telescope is affected both by scattering and absorption effects, all of them depending on the altitude of the starting point. These effects come from the Rayleigh scattering by the air molecules (dominant in high atmosphere and for low wavelength values, mainly acting on Čerenkov light reflected from ground), the Mie scattering by aerosols (dust, smoke,...) and droplets (clouds), and from the absorption by ozone (rather transparent for large wavelength values, influences Cerenkov photons more than fluorescence ones). In the EUSO chain we used the LOWTRAN7 software package [20] for different atmospheric models, and basically the US Standard Atmosphere 1976 and Supplements [21], and NRLMSISE-00 [22] models.

Clouds have a prior effect in EUSO; in fact they can hide the signal (fluorescence and/or Čerenkov), hide the maximum of fluorescence, affect the proportionality between detected signal and shower energy [23]. In the EUSO chain, top height and optical depth are currently derived from meteorological databases as ISCCP [24] and TOVs [25]. At the EUSO operation time, this basic info will be directly derived from the EUSO atmosphere sounding devices (Lidar and IR-camera).

Background must be taken into account, too. In fact, looking at Nadir, EUSO will be sensitive to the light coming from the outer Space and upward reflected through Earth albedo, as well as to any source of light located in upper atmosphere below its altitude location $(\sim 400 \mathrm{~km})$. The level of background depends on various parameters as moon phase, geographical position, seasonal and meteorological conditions. In the EUSO chain, the level of background is programmable around its "natural" reference level (see Reference [8]).

The simulation of the main EECR/v detector describes the full path of a photon from the optics pupil to the read-out electronics. The shower will appear as a single track event (embedded in the background) whose duration, position and intensity are related to the arrival direction, energy and nature of the Primary EECR/v. The space-time image is given in terms of $X-T$ and $Y-T$ projections of the collected photoelectrons, $X$ and $Y$ being the coordinates inside the field of view; the time coordinate $\mathrm{T}$ measures the shower development in depth, providing info about the shower length in the third direction, the height in the atmosphere. In Fig.4 a rough example of simulated event is given.

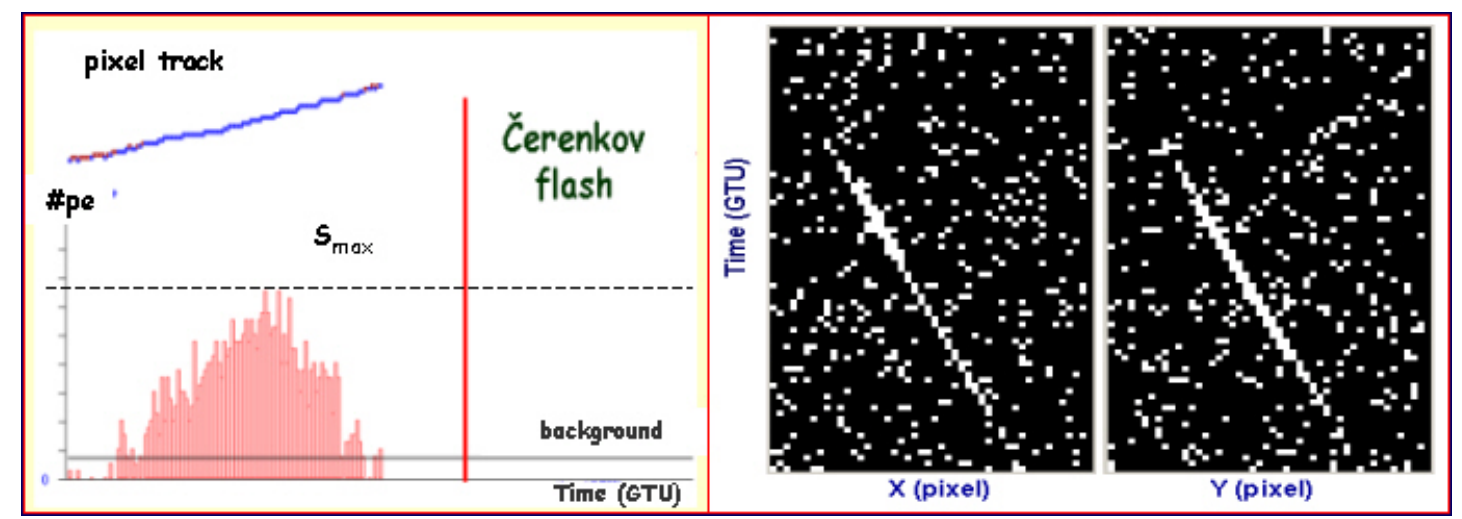

FIGURE 4: Simplified example of a simulated EECR event $\left(5 \times 10^{20} \mathrm{eV}, 60^{\circ}\right.$ arrival zenith angle) as seen by EUSO. Left panel: time profile of photo-electrons (pe). The shower size at its maximum development, $S_{\max }$, is related to the EECR energy. Right panel: schematic view of the track along the two projection views (as registered by the read-out electronics). Čerenkov flash is

not here represented. The Gate Time Unit, GTU, is the basic time unit for counting photoelectrons in an EUSO pixel.

Finally, the reconstruction of the event requires specific algorithms: dedicated pattern recognition and fitting techniques to extract the signal track from the background and to derive its arrival direction [26, 27, 28]; reconstruction of the maximum shower size and of the depth where it occurs, estimate of the track integral and shower elongation, all of them necessary to derive the energy of the Primary and the expected elemental composition resolution power [29]. 
For what concerns event reconstruction and scientific data analysis, a particular effort is being devoted to the deconvolution algorithm to take into account the distortions introduced by the variability of the atmospheric condition as seen from EUSO on the ISS. To reach this goal, housekeeping, engineering, ancillary and atmosphere sounding data are required to apply the necessary gain, attitude and atmospheric corrections. Part of this information will be measured on-board; nevertheless, external distributed information (atmospheric, meteorological, geographic databases and maps, and more) would be necessary to complete the knowledge of the observation conditions. Moreover, in a real operation phase, astrophysical catalogues could be used to correlate the reconstructed EECR/v real events seen by EUSO with astronomical phenomena, to help in the interpretation of their origin and potential source.

In summary, different algorithms as well as different heterogeneous information and data are necessary to simulate the physical process and the EUSO behaviour, to optimise its design, to calibrate its response function, to reconstruct the event and then to properly analyse and certify EUSO observational data.

\section{ESAF - EUSO SIMULATION AND ANALYSIS FRAMEWORK}

The EUSO Collaboration includes scientists coming from different disciplines (astrophysics, physics of particles, atmosphere science) and using different software systems, too; even in this sense, EUSO is a challenge.

During the EUSO Phase A study, a set of parallel s/w modules were built to perform a fast End-To-End simulation chain finalized to the detector design and optimisation. Only part of the involved variables were used. At the same time, a project started to define a sophisticated software framework on which to develop a complete and coherent data simulation, reconstruction and analysis system: its name is ESAF.

ESAF stands for "EUSO Simulation and Analysis Framework" [10, 11]. It is an integrated software designed to handle the event simulation chain (shower development simulation, light production due to fluorescence and Čerenkov, atmospheric effects, light transport to the EUSO main detector, response of the EUSO detector itself) and the reconstruction and analysis of both simulated and real events.

The design of ESAF is based on the ROOT package [30]. Taking into account the different disciplines which can be interested in EUSO, the time on which EUSO will operate (its launch is foreseen in 2010) and the current developments in software logic and programming, the choice of a ROOT-based system seems to be the most suitable one: ROOT is an Object Oriented (OO) programming, it is implemented on various platforms (Linux, Windows, ...), presents interfaces towards standard formats as FITS and IDL, has been adopted for some specific tasks in ground-based observatories and space-missions in high energy physics and astrophysics (ex. HESS, INTEGRAL), provides basic graphics tools as well as tools for automatic documentation generation.

ESAF is written in C++ language; it is currently implemented on Linux and requires, as compiler, the standard "gcc" version 3.2 or higher [31]. ESAF is built with an "onion-like" structure: outer layers are more general and abstract than the inner ones which becomes step by step more and more specialised. This is accomplished with a massive use of abstract interfaces and "factories". In this way outer layers need to know only the interface of objects in the inner layers (what these objects do) without knowing what the actual implementation will be.

ESAF is able to handle external packages, even if written in different languages as Fortran: for example, the event/shower generators UNISIM and SLAST are already part of ESAF, while the interface to CORSIKA and AIRES are under development as well as the implementation of LOWTRAN for light transmission in atmosphere.

Most of the ESAF behaviour is controlled by variables which values are settled in several configuration files: a set of such files, referring to well-defined standard configurations, forms a database from which user can choose the proper one as a starting base, and so change it in only few variable values.

The detector simulation package inside ESAF has been completely written from scratch. It includes a simulation that follows individual photons from the entrance at the first lens of the optics and track them up to the photocathode of each single PMT. Shape, filling, position in space of the whole object of the focal surface are simulated in ESAF, as well as the whole signal treatment by the electronics.

As schematically shown in Fig.5, referring to the simulation part, the top level of the ESAF onion-like structure is "EusoApplication" which acts as interconnection between the various modules (blue boxes). The three main modules on the left (ShowerSource, LightSource, RadiativeTransfer) refer to the simulation of all the physical 
phenomena occurring outside EUSO that can produce photons on the entrance of the detector (extensive air showers, lightning, meteors,... ). Atmosphere plays here its twofold role: light emission and transmission medium. The simulation of the main EECR/v telescope is performed in the EusoDetector box and along its Optics, FocalPlane and Electronics sub-modules (IR camera and Lidar-based system are not here considered yet). All the modules are implemented as objects that are instances of C++ classes; it is therefore easy to get many different versions of each module, but preserving their interface. The simulation of the physical process, which can be done in several ways, will provide, as output, a list of photons on the entrance of the EECR/v telescope: EusoApplication does not know how the photons are produced and propagated, it only knows that there exists an object that can give it the list of photons. The EusoDetector module then handles each photon in the list propagating it through the detector from the Optics up to the Electronics that produces a Telemetry object. ESAF provides two kinds of files as output of its simulation part: a ROOT file with the description of the events and the detector configuration, and a compressed ASCII "telemetry" file that mimics the real situation during the operative life of EUSO, i.e, what it is expected to arrive to the ground station from the EUSO accommodated on the ISS.

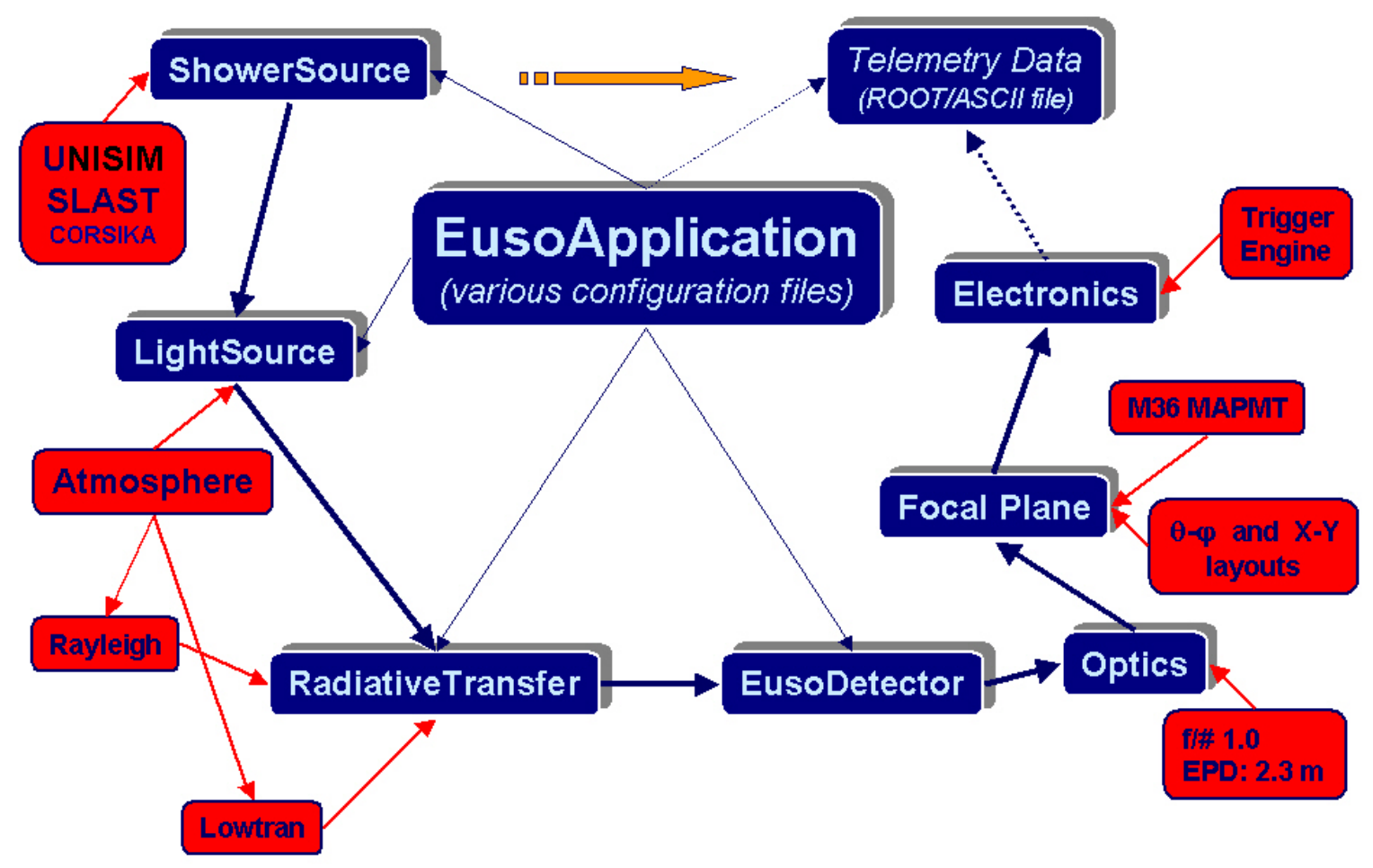

FIGURE 5: Top level structure of the ESAF "simulation" component

The Object Oriented programming used in ESAF allows to reach a high modularity and it is then possible to run single parts of the ESAF chain and to check quantitatively the differences between various configurations of the detector or different approximations applied in the simulation of the physical processes involved in. Each step can run individually and independently from the other ones, and it will be therefore possible to run the same reconstruction and analysis code for both simulated and real data.

A sketch of the general structure proposed for the ESAF reconstruction part is shown in Fig.6. Input data comes either from the simulation module (ROOT file) or from the real data stream (pre-processed telemetry file). Their reading is handled by the blue box named InputModule. The next module, RecoEvent, acts as the container structure for the input event to be reconstructed; it allows the access to several information from the event header to the trigger and readout at pixel level. The RecoFramework box is the main structure which build the chain of modules proper of the ESAF reconstruction component. Different tasks of reconstruction can be present (arrival direction, energy, ...) and different algorithms can be implemented for each task. As described above, even in the reconstruction part each step can run individually, replaced, excluded, combined and compared. A major role is played once a time by the configuration files which will include also what modules have to be applied during the event reconstruction step. Moreover, a specific AnglePixelMap module is present to define what channel in EUSO corresponds to a given arrival angle on its focal surface; this is necessary to perform the first step of the event geometrical reconstruction. 


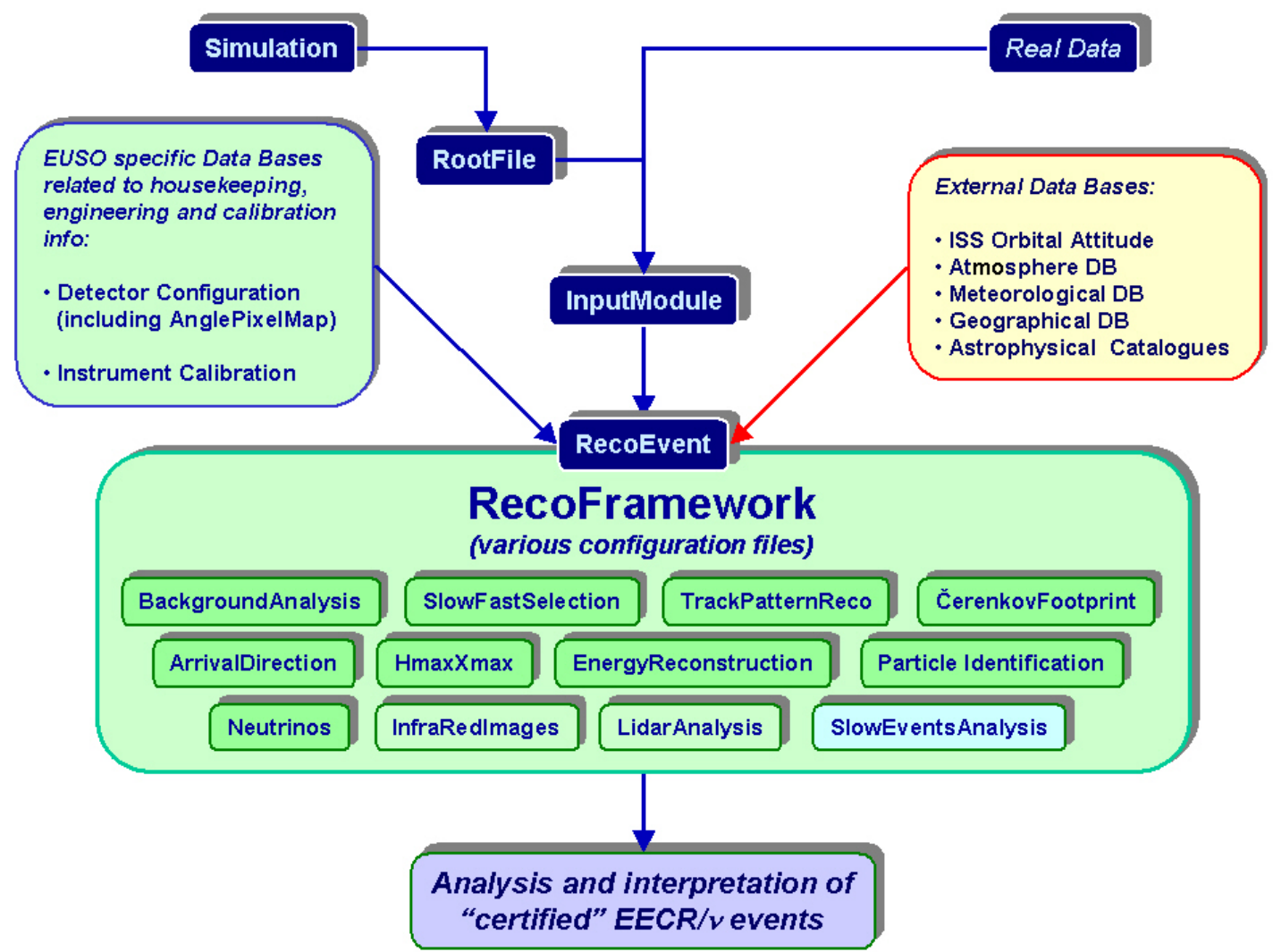

FIGURE 6: General structure of the ESAF "reconstruction" component

The ESAF code makes use of the CVS Concurrent Version System [32] and resides at the CVS Server in Lyon, France [33]. The current status of ESAF implementation is briefly shown in Fig.7.
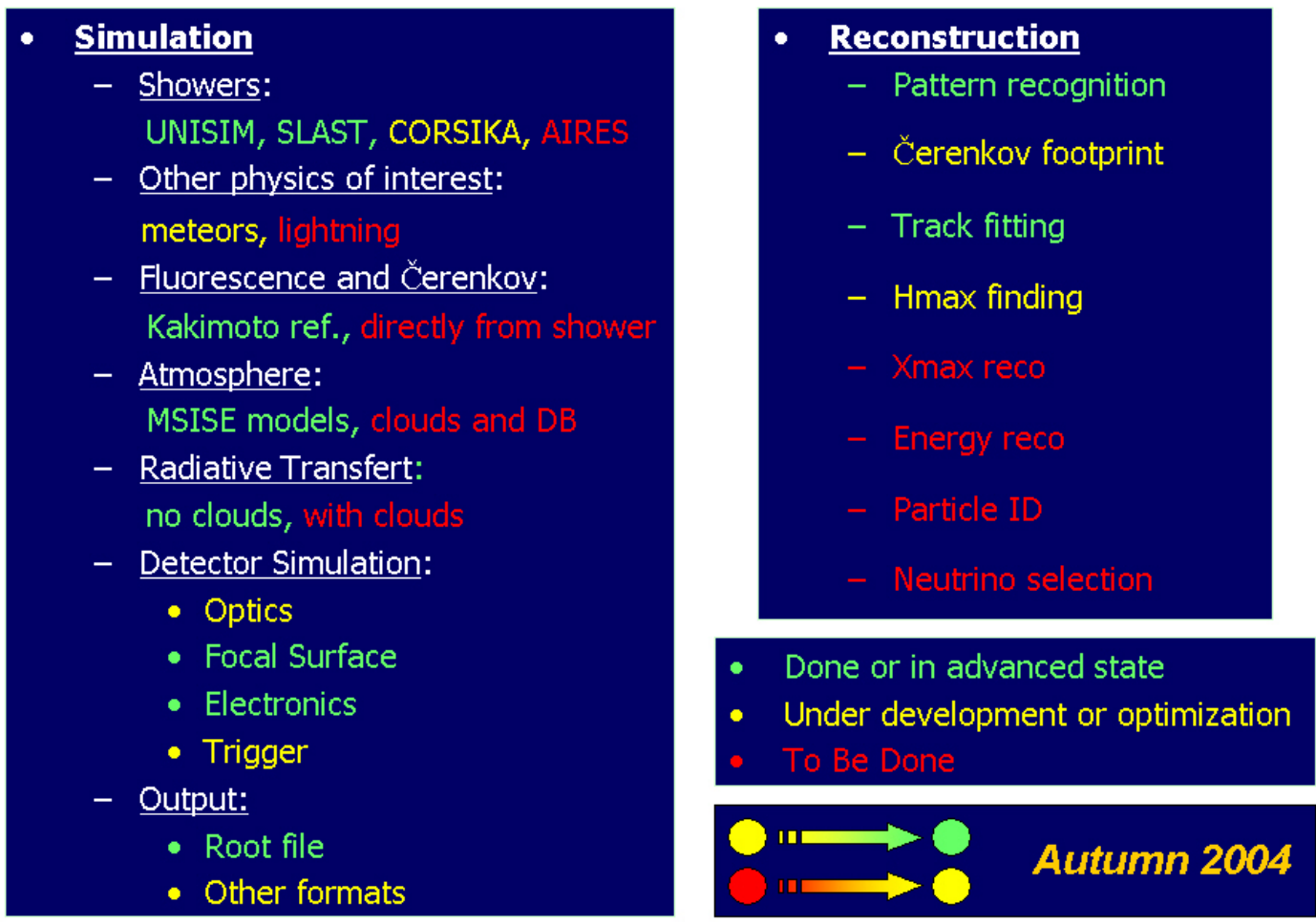

FIGURE 7: Status of ESAF implementation 


\section{CONCLUSIONS}

The Extreme Universe Space Observatory EUSO will do astronomy looking downward the dark Earth atmosphere from the International Space Station.

EUSO is the first Space mission devoted to the investigation of EECR/v using the Earth atmosphere as a giant detector. The peculiarities of the EUSO observational approach require a dedicated effort for the evaluation of the expected features of the detected signals and for their reconstruction, even for what concerns the deconvolution algorithms necessary to estimate the expected shower longitudinal profile, with reference to the distortion introduced by the variability of the atmosphere conditions as seen from EUSO on the ISS.

A complete software infrastructure named ESAF, EUSO Simulation \& Analysis Framework, is under development suitable for the overall simulation and analysis chain. A first version of ESAF has been delivered in Spring 2004; an updated version is foreseen within Autumn 2004.

\section{REFERENCES}

[1] Baltrusaitis R.M., et al. (1985) NIM A, 311, 338

[2] HiRes Home Page http://hires.physics.utah.edu

[3] Auger Home Page http://www.auger.org

[4] Bird D.J., et al. (1993) Phys. Rev. Lett. 71, 71

[5] Linsley J. (1979) SOCRAS, proposal in response to Astronomy Survey Committee

[6] Catalano O. (2001) II Nuovo Cimento 24-C, 3, 445-470

[7] Scarsi L. (2001) II Nuovo Cimento 24-C, 4-5, 471-482

[8] EUSO Collaboration (2004), "EUSO Instrument - Report on Phase A Study", EUSO-PI-REP-005, 21 apr04

[9] EUSO Home Page http://www.euso-mission.org

[10] De Marco D. and Pallavicini M. (2002) "ESAF", EUSO-SDA-REP-008, 13 May 2002

[11] Pallavicini M. and Thea A. (2004) "ESAF Users Guide", EUSO-SDA-REP-014, 26 February 2004

[12] Maccarone M.C. and Mineo T. (2001) Procs. 27th ICRC, Hamburg, Germany, Vol.HE, pp.852 -855

[13] Colin P. (2003) "Background Sources", EUSO-SIM-REP-006, 6 February 2003

[14] Heck D. et al. (1998) Report FZKA6019, Forschungszentrum Karlsruhe

[15] Sciutto S. (1999) Auger Project Report GAP-99-044

[16] Bottai S. (2001), Proc. 27th ICRC, Hamburg, Vol.HE

[17] Linsley J. (2001), Procs. 27th ICRC, Hamburg, Germany, Vol.HE, 502 \& 683

[18] Catalano O., Maccarone M.C., Sacco B., Scarsi L. (2001), Procs. 27th ICRC, Hamburg, D, Vol.HE, $835-838$

[19] Naumov D. (2003) "SLAST", EUSO-SDA-REP-015, 13 March 2003

[20] Kneizys F.X. et al. (1996) "The MODTRAN 2/3 Report and LOWTRAN 7 MODEL", Abreu \&. Anderson Eds.

[21] USSA-76, US Standard Atmosphere http://nssdc.gsfc.nasa.gov/space/model/atmos/us standard.html

[22] NRLMSISE-00 Model 2001 http://nssdc.gsfc.nasa.gov/space/model/atmos/nrlmsise00.html

[23] D'Alì Staiti G., Bottai S., Plagnol E., Mitev V. (2004) "End to End Simulation”, EUSO-SIM-REP-013, $16 a p r 04$

[24] ISCCP, International Satellite Cloud Climatology Project, http://isccp.giss.nasa.gov

[25] TOVS, Tiros Operational Vertical Sounder, http://www.ozonelayer.noaa.gov/action/tovs.htm

[26] Maccarone M.C., Catalano O., Giarrusso S., Sacco B. (1999) Proc. 26th ICRC, Vol.5, 445

[27] Bottai S. (2002) "Some algorithms for direction reconstruction in EUSO", EUSO-SDA-REP-006, 10 June 2002

[28] Colin P., Naumov D., Nedelec P. (2003) "EUSO Reconstruction Algorithms", EUSO-SDA-REP-016, 13mar03

[29] EUSO Collaboration (2003), various contributions in Proc. 28th ICRC, Tsukuba, Japan, Vol. HE

[30] The ROOT System http://root.cern.ch

[31] GNU Compiler Collection http://gcc.gnu.org

[32] CVS, Concurrent Version System http://www.cvshome.org

[33] Service CVS au Centre de Calcul de l'IN2P3 http://cvs.in2p3.fr 